\title{
The impact of Bacille Calmette-Guerin shortage on immunisation practice and policies in Europe - A Paediatric Tuberculosis Network European Trials Group (ptbnet) survey
}

\section{Kontturi, Antti}

2016-12

Kontturi , A , Santiago , B , Tebruegge , M , von Both , U , Salo , E \& Ritz , N 2016 , ' The impact of Bacille Calmette-Guerin shortage on immunisation practice and policies in Europe - A Paediatric Tuberculosis Network European Trials Group (ptbnet) survey ' , Tuberculosis , vol. 101 , pp. 125-129 . https://doi.org/10.1016/j.tube.2016.08.005

http://hdl.handle.net/10138/229901

https://doi.org/10.1016/j.tube.2016.08.005

publishedVersion

Downloaded from Helda, University of Helsinki institutional repository.

This is an electronic reprint of the original article.

This reprint may differ from the original in pagination and typographic detail.

Please cite the original version. 
EPIDEMIOLOGY

\title{
The impact of Bacille Calmette-Guérin shortage on immunisation practice and policies in Europe - A Paediatric Tuberculosis Network European Trials Group (ptbnet) survey
}

\author{
Antti Kontturi a, b, Begoña Santiago c, Marc Tebruegge ${ }^{\mathrm{d}, \mathrm{e}, \mathrm{f}}$, Ulrich von Both ${ }^{\mathrm{g}, \mathrm{h}}$, \\ Eeva Salo ${ }^{\mathrm{a}}$, Nicole Ritz ${ }^{\mathrm{f}, \mathrm{i}}$, , Ptbnet BCG shortage project collaborators, on behalf of the \\ Paediatric Tuberculosis Network European Trials Group (ptbnet) \\ a Department of Paediatrics, Children's Hospital, University of Helsinki and Helsinki University Central Hospital, Helsinki, Finland \\ b Clinicum, Department of Public Health, Faculty of Medicine, Doctoral Programme in Population Health, University of Helsinki, Finland \\ c Sección Enfermedades Infecciosas Pediátricas, Servicio de Pediatría, Hospital General Universitario Gregorio Marañón, Instituto de Investigación Sanitaria \\ Gregorio Marañón (IiSGM), Madrid, Spain \\ d Faculty of Medicine \& Global Health Research Institute, University of Southampton, Southampton, UK \\ e Department of Paediatric Immunology and Infectious Diseases \& NIHR Respiratory Biomedical Research Unit, University Hospital Southampton NHS \\ Foundation Trust, Southampton, UK \\ ${ }^{\mathrm{f}}$ Department of Paediatrics, The University of Melbourne, Parkville, Australia \\ ${ }^{g}$ Division of Paediatric Infectious Diseases, Dr von Hauner Children's Hospital, LMU, Munich, Germany \\ ${ }^{\mathrm{h}}$ German Centre for Infection Research (DZIF), Germany \\ ${ }^{1}$ University Children's Hospital Basel, Paediatric Infectious Diseases, Vaccinology, and Paediatric Pharmacology, Basel, Switzerland
}

\section{A R T I C L E I N F O}

\section{Article history:}

Received 1 June 2016

Received in revised form 23 August 2016

Accepted 25 August 2016

\section{Keywords:}

Tuberculosis

TB

BCG

Supply

Public health

Vaccine

\section{S U M M A R Y}

Background: Recent reports indicate an ongoing BCG shortage that may influence immunisation practice. This study aimed to determine current availability of BCG vaccine across Europe, and implications on immunisation practices and policies in Europe.

Methods: Web-based survey among Paediatric Tuberculosis Network European Trials Group (ptbnet) members, between May and October 2015.

Results: Twenty individuals from 13 European countries participated. Ongoing shortages were reported in eight countries routinely using BCG $(8 / 11,73 \%)$. As a consequence of the shortage, BCG was not given as completely unavailable in some countries $(2 / 8,25 \%)$, was given only whenever available $(1 / 8,13 \%)$, or only in certain regions of the country $(1 / 8,13 \%)$. Strategies reported to reduce loss of immunisation were administration to selected high-risk individuals $(2 / 8,25 \%)$, or cohorting vaccinees on specific days to maximise the use of multi-dose vials $(3 / 8,38 \%)$. Authorities in two countries each were considering a change of manufacturer/supplier $(2 / 8,25 \%)$.

Conclusions: The BCG shortage in Europe leads to significant changes in immunisation policies including changes of BCG vaccine strain and manufacturer. In addition, infants and children eligible for immunisation are at risk of not receiving BCG. To ensure necessary BCG immunisations, collaboration between national health agencies and vaccine manufacturers is crucial.

๑) 2016 Elsevier Ltd. All rights reserved.

Abbreviations: AE, adverse event; BCG, Bacille Calmette-Guérin; ECDC, European Centre for Disease Prevention and Control (the); TB, tuberculosis; MDR, multidrug-

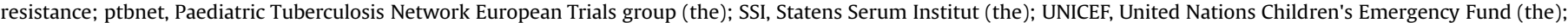
WHO, World Health Organization (the); XDR, extensive drug resistance.

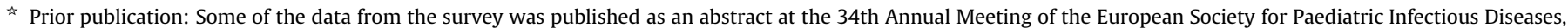
2016.

* Corresponding author. Infectious Diseases and Paediatric Pharmacology, University Children's Hospital Basel, Spitalstrasse 33, CH-4031 Basel, Switzerland.

E-mail addresses: antti.kontturi@helsinki.fi (A. Kontturi), bsantiagogarcia@gmail.com (B.Santiago), m.tebruegge@soton.ac.uk (M. Tebruegge), uvb9@gmx.de (U. von Both), eeva.salo@hus.fi (E. Salo), nicole.ritz@unibas.ch (N. Ritz). 


\section{Introduction}

Globally, tuberculosis (TB) is one of the most important infectious diseases and children are especially vulnerable [1,2]. Compared to adults, children infected with Mycobacterium tuberculosis are more likely to develop active TB disease within a short period of time. In 2014, an estimated 1 million new TB cases and 140.000 TB deaths occurred globally among children below 15 years of age [1]. Although the overall TB incidence in Europe is decreasing, TB remains a major public health concern [3]. The World Health Organization (WHO) estimates that there were 31.000 new TB cases among children in the European Region in 2014 , of which only $32 \%$ were reported [1]. In many countries, the majority of TB cases are foreign-origin, and with the current migration in Europe this will affect epidemiology in low incidence TB countries [3,4]. Furthermore, with the emergence of multidrugresistant (MDR) and extensively drug-resistant (XDR) TB, treatment is becoming increasingly challenging, placing further emphasis on the importance of prevention [3].

Infant Bacille Calmette-Guérin (BCG) immunisation prevents severe and disseminated TB in children, and may also protect against latent TB infection [5,6]. The protective efficacy of BCG against drug-susceptible and MDR TB is likely similar and, therefore, BCG immunisation has been suggested as a preventative measure for individuals exposed to MDR-TB [7]. BCG is one of the most commonly used vaccines globally, with approximately $90 \%$ of children covered worldwide and over 120 million individuals vaccinated annually $[8,9]$. Vaccine coverage is however not uniform worldwide since many low TB incidence countries do not offer BCG at all or only for selected high-risk groups, while some high TB incidence countries including Papua New Guinea, Somalia and the Ukraine have coverage rates below 70\% [10]. In high TB incidence countries, BCG immunisation is a cost-effective intervention and an essential part of TB control and prevention particularly in young children [5]. In addition, BCG immunisation is known to have a number of non-specific or heterologous effects, including reducing all-cause mortality in resource limited countries and influencing the immune response to other routine immunisations [11,12].

Currently, three BCG vaccine strains are in large-scale production (BCG Denmark, BCG Russia and BCG Japan) which are produced by five WHO prequalified vaccine manufacturers in Bulgaria, Denmark, India and Japan $[13,14]$. In Europe, BCG Denmark has been the most commonly used BCG vaccine strain in the last decade $[15,16]$.

BCG supply and demand has been influenced by a decrease in manufacturers worldwide together with an increased global demand, likely as a result of rising birth rates and improved immunisation coverage. In 2014, the demand increased by more than 20 million doses, as a result of unmet demand carry-over from the previous year and incremental demand from some large countries in the Middle East and South East Asia, and it is estimated to increase further by an annual 10 million doses [8]. In addition to this, since 2013, a reduced production capacity of two manufacturers, the Statens Serum Institut (SSI) in Denmark and the Serum Institute of India, further affects annual global BCG availability [17]. Although some manufacturers were able to increase their production, the overall BCG availability fell by 6 million doses [8]. In fact, the United Nations Children's Emergency Fund (UNICEF) estimated that in 2014 the global BCG production fell short by over 20 million doses, and that in 2015 the unmet demand carry-over together with growing demand and continuing production shortage may result in a deficit of over 70 million doses [8].

This shortage is likely to affect BCG immunisation practices and coverage in Europe, as universal or selective immunisation is a part of TB prevention in at least 27 European countries [15]. We therefore conducted a survey to determine current availability of BCG vaccine across Europe, and describe the implications on immunisation practices and policies.

\section{Methods}

We conducted a web-based survey among Paediatric Tuberculosis Network European Trials Group (ptbnet) members between May 2015 and October 2015. Ptbnet comprises clinicians, microbiologists, epidemiologists and researches with a special interest in paediatric TB $[18,19]$. At the time of the survey, ptbnet had a total of 117 members in 25 European countries. Members were invited by e-mail to participate in the survey, which included 16 questions on the participant's demographics, use of BCG vaccine, BCG availability, and consequences of possible shortage. Answers were collected through Google Docs software (Google Inc., Mountain View, CA, US) and/or Word questionnaire (Microsoft Corporation, Redmond, WA, US). Participants were allowed to reply to all or only some of the questions. The full questionnaire and the response rates to each question are shown in the supplementary data. Two reminders were sent: to all members in September 2015, and only to the designated ptbnet representative of member countries in October 2015. In instances where responses were unclear or conflicting, these were clarified through email until January 2016. The final data were collated into and analysed using Excel (Version 2011, Microsoft Corporation, Redmond, WA, US).

\section{Results}

Twenty individuals (20/117, 17\%) from 13 European countries (13/25, 52\%) participated (Table 1). Respondents were based in Austria, Belgium, Bulgaria, Croatia, Finland, Germany, Greece, Italy, Slovenia, Spain, Sweden, Switzerland and the United Kingdom.

Respondents from eleven countries reported using BCG as part of their national immunisation programme (11/13, 85\%). The most commonly reported vaccine strain used was BCG Denmark SSI 1331 (10/11, 91\%). In addition, BCG Russia Bulbio Sofia was used, in Bulgaria (1/11, 9\%), where it is manufactured. BCG was reported to be imported in most countries $(10 / 11,91 \%)$, with the exception of Bulgaria, where it was reported to be produced nationally (1/11, $9 \%$ ). Respondents reported that BCG was distributed by government agencies or departments of health in most countries $(5 / 7$, $71 \%$ ); in two countries, private companies were reported to be national distributors - Econophar (Kortenberg, Belgium) and Pro Farma AG (Baar, Switzerland). Data regarding the total number of doses imported in 2014 were only available from two countries and therefore not further analysed.

Specific national BCG immunization policies that were reported included selective newborn immunization (5/11, 45\%), selective immunisation at a later age $(3 / 11,27 \%)$, or both $(1 / 11,9 \%)$, and universal newborn immunisation (2/11, 18\%). BCG immunisations were reported to take place in maternity hospitals $(3 / 11,27 \%)$, community health centre in primary care $(3 / 11,27 \%)$, primary school (1/11, $9 \%)$, or all of these/multiple places $(4 / 11,36 \%)$.

A current BCG shortage was reported by respondents from eight countries $(8 / 11,73 \%)$. In addition, a respondent from Croatia reported a recent shortage that lasted less than a month; the respondent stated that unmet carry-over immunisations took place when the shortage had resolved. All affected countries were using BCG Denmark. Responses concerning the onset of the shortage varied and were sometimes conflicting; most respondents reported that the shortage had started at some point from 2013 onwards.

Changes in BCG immunisation practices or policies were reported by respondents from seven countries (7/8, 86\%) (Table 2). Members from Belgium and Italy reported a discontinuation of 
immunisations as a result of BCG being entirely unavailable; in Greece, BCG was reported to be given whenever available. In Spain, BCG was reported to be available only in certain regions of the country and, due to the shortage, given only to selected highrisk individuals. Members from Finland, Sweden and the United Kingdom reported cohorting vaccinees on specific days to use as many doses from multi-dose vials as possible. One respondent from the United Kingdom also reported that due to the shortage BCG was only given to selected high-risk individuals. Switching manufacturer and BCG strain were reported to be under consideration in Belgium and Finland. A respondent from Sweden reported that national data about the BCG shortage was being collected. Out of the eight countries in which a BCG shortage was reported, five reported that national BCG stockpiling was not taking place $(5 / 8,63 \%)$, two reported that stockpiling was done (2/ $8,25 \%)$, and one did not respond to this question. Public national information about the BCG shortage was reported to be available in five countries $(5 / 8,63 \%)$, and unavailable in three countries ( $3 /$ $8,38 \%)$.

Shortage of BCG for bladder cancer treatment was reported by respondents from two countries $(2 / 11,18 \%)$ and nine respondents had no personal knowledge on this matter.

\section{Discussion}

Our results show that the ongoing global BCG vaccine shortage has significant consequences on immunisation practice in Europe. The survey identified BCG shortages in eight European countries, all of which were using BCG Denmark produced by SSI. A survey conducted in 2013 showed that 24 out of 31 countries in the European region were using BCG Denmark, and that at least 3 out of the 18 high priority countries in the fight against TB, as defined by the European Centre for Disease Prevention and Control (ECDC), were using BCG Denmark $[3,15]$. Therefore, it is likely that the limited supply of BCG Denmark affects also further countries not captured in our survey. Among the countries captured in our survey, only Bulgaria is classified as a high priority country in the fight against TB [3]. Bulgaria uses a nationally produced BCG vaccine, and no shortage was reported from this country.

Our results also highlight that in many European countries ongoing BCG shortages have resulted in changes in immunisation practices and policies. One approach is optimising the use of multidose BCG vaccine vials by centralising and cohorting BCG immunisations to reduce vaccine loss, and there is some data to support that this may be effective. Unpublished data from Finland suggest that centralising immunisations and giving BCG only on specific dates using multidose vials resulted in halved vaccine consumption (personal communication from Pertti Sormunen, Director of Pharmaceutical Wholesale, National Institute for Health and Welfare, Finland, April 19, 2016). However, a report from Australia where a similar approach was used, estimated vaccine wastage to be around $75 \%[20]$.

Table 1

Demographic details of participants and summary of BCG use and shortage.

\begin{tabular}{lll}
\hline & $\mathrm{n}$ & $\%$ \\
Participants working at & 20 & 100 \\
Paediatric hospital & 16 & 80 \\
General hospital & 3 & 15 \\
Outpatient Tb specialised clinic & 1 & 5 \\
Countries & 13 & 100 \\
Not routinely using BCG (Austria, Germany) & 2 & 10 \\
No current shortage (Bulgaria, Slovenia, Croatia*) & 3 & 15 \\
Current shortage (Belgium, Finland, Greece, Italy, & 8 & 75 \\
Spain, Sweden, Switzerland, United Kingdom) & & \\
\hline
\end{tabular}

${ }^{*}$ Reported a recent shortage prior to the survey being conducted.

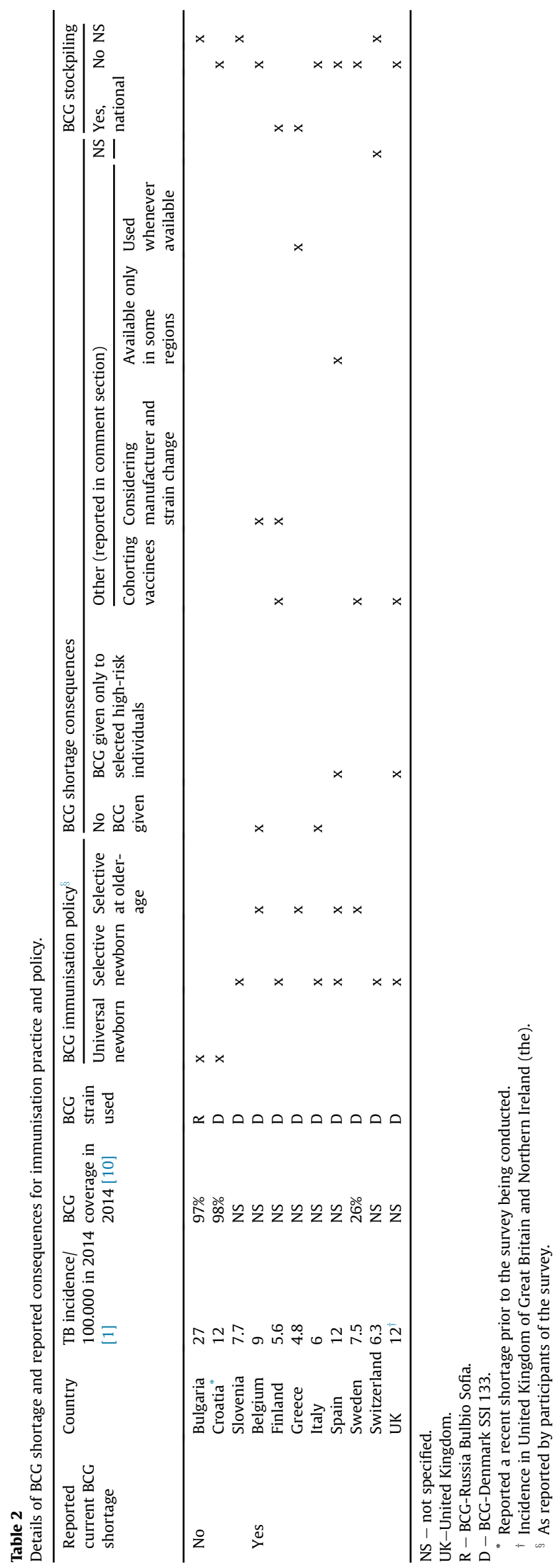


Another option to address the ongoing shortage is changing the BCG vaccine manufacturer. In our survey, authorities in two countries (Belgium and Finland) were reported to be considering an alternative manufacturer. As of April 2016, two countries captured in the survey (Finland and Sweden) have changed the manufacturer and are now using BCG-Japan (personal communication from Pertti Sormunen, Director of Pharmaceutical Wholesale, National Institute for Health and Welfare, Finland, April 19, 2016) [21]. Furthermore, other countries, including France and Australia, have temporarily replaced BCG Denmark with BCG Brazil manufactured by Biomed Lublin in Poland [22,23]. This decision is somewhat surprising as there is limited information on safety and protective efficacy of this BCG vaccine strain as it accounts for only a very small proportion of BCG vaccine strains used globally [24]. BCG Poland is a descendant of BCG Brazil (frequently called BCG Moreau or BCG Rio de Janeiro) which has been used in Poland exclusively since 1955 and has been shown to be genetically different to its parent strain [24]. Recent update from Public Health England reports that the shortage is on-going [25] Considering the ongoing BCG Denmark vaccine delivery delays, it appears likely that further countries in Europe and globally will change to another BCG manufacturer, resulting in a change of the BCG vaccine strain used in their national immunisation programmes.

Importantly, changes in the BCG vaccine strains may have significant consequences. Different BCG vaccine strains have been shown to influence the immune response and protection against TB $[9,14,26]$. Recent evidence from a study comparing the genomes and transcriptomes of several BCG vaccine strains showed an increase in expression of ribosomal proteins in some strains which may be responsible for increased immunogenicity and better protection against TB [27]. However, it currently remains unclear which BCG vaccine strain offers the best protective efficacy, and consequently any change might affect protection on a population level. In addition, previous reports have shown that changes in the $B C G$ vaccine strain can be associated with an increased incidence of BCG-associated adverse events (AEs). For instance, in 2002 Finland changed from BCG Glaxo to BCG Denmark, as the production of the former was discontinued. In the subsequent two years the incidence of BCG-associated AEs increased considerably, which, in combination with a decreasing TB incidence, ultimately led to discontinuation of universal BCG immunisation in Finland in 2006 $[28,29]$. Similarly, a change from BCG Connaught to BCG Denmark in Australia in 2012 led to, when administered doses were taken into account, an estimated increased incidence of BCG-induced abscesses and lymphadenitis [20]. AEs related to immunisations cause concern in both healthcare professionals and the general public, potentially impacting the general attitude on immunisations and thereby influencing coverage rates. Thus, monitoring protective efficacy and AEs after a BCG vaccine strain change is of utmost importance.

The key limitations of this survey are the relatively small number of participants, and the fact that we were unable to obtain data from several European countries. In addition, the majority of participants were based in specialised paediatric institutions, and the data may therefore not be representative of the situation in regional hospitals or at the private practice level. As the ptbnet is mainly a European network we were unable to collect data on BCG vaccine shortage outside Europe, where the impact is likely to be considerably greater. However, a report from the WHO Child TB Subgroup meeting in December 2015 stated that critical BCG vaccine shortages were affecting many countries worldwide [17].

It appears obvious that the problems related to the ongoing BCG vaccine shortages cannot be solved by changing the vaccine manufacturer, as this approach simply shifts the demand to another manufacturer. It is therefore important that supranational health organisations, such as UNICEF, WHO and ECDC, engage with vaccine manufacturers to jointly devise mechanisms that ensure a stable BCG vaccine supply that meets global demand. Notably, in December 2015 UNICEF reported having increased orders with three manufacturers amounting to an additional 38.3 million BCG doses in 2015, which still left an estimated deficit of 16.5 million doses that year [30]. To our knowledge there is currently no specific national or global assessment of the impact of this deficit on childhood TB, and such an assessment should be conducted by either WHO or UNICEF based on TB notification rates in the next few years. In addition, the communication between manufacturers, national public health agencies and end users of the BCG vaccine requires improvements so that the immunisation services can adapt timely in the event of future shortages.

\section{Funding}

None.

\section{Competing interests}

None declared.

\section{Ethical approval}

Not required.

\section{Acknowledgements}

The following authors Daniel Blázquez, Matthias Bogyi, Danilo Buonsenso, Alexandra Dreesman, Uros Krivec, Françoise Mouchet, Sahar Nejat, Olaf Neth, Ivan Pavić, Ezia Ruga, Rupert Schlags, Antoni Soriano-Arandes, Steffi Thee, Maria Tsolia and Svetlana Velizarova are supported for this work.

\section{Appendix A. Supplementary data}

Supplementary data related to this article can be found at http:// dx.doi.org/10.1016/j.tube.2016.08.005.

\section{References}

[1] Global tuberculosis report 2015. 20th ed. World Health Organization; 2015.

[2] Perez-Velez CM, Marais BJ. Tuberculosis in children. N Engl J Med 2012;367: 348-61.

[3] European Centre for Disease Prevention and Control/WHO Regional Office for Europe. Tuberculosis surveillance and monitoring in Europe 2015. Stockholm: European Centre for Disease Prevention and Control; 2015.

[4] Ritz N, Brinkmann F, Santiago Garcia B, Tebruegge M, Kampmann B. Paediatric tuberculosis network European trials g. Tuberculosis in young refugees. Lancet 2015;386:2475-6.

[5] Trunz BB, Fine PEM, Dye C. Effect of BCG vaccination on childhood tuberculous meningitis and miliary tuberculosis worldwide: a meta-analysis and assessment of cost-effectiveness. Lancet 2006;367:1173-80.

[6] Soysal A, Millington KA, Bakir M, Dosanjh D, Aslan Y, Deeks JJ, Efe S, Staveley I, Ewer K, Lalvani A. Effect of BCG vaccination on risk of Mycobacterium tuberculosis infection in children with household tuberculosis contact: a prospective community-based study. Lancet 2005;366:1443-51.

[7] Seaworth BJ, Armitige LY, Aronson NE, Hoft DF, Fleenor ME, Gardner AF Harris DA, Stricof RL, Nardell EA. Multidrug-resistant tuberculosis. Recommendations for reducing risk during travel for healthcare and humanitarian work. Ann Am Thorac Soc 2014;11:286-95.

[8] United Nations Children's Fund. BCG vaccine: current supply \& demand outlook. UNICEF Supply Division; 2014. available from: http://www.unicef org/supply/files/BCG_Supply_Status_December_2014.pdf.

[9] Ritz N, Hanekom WA, Robins-Browne R, Britton WJ, Curtis N. Influence of BCG vaccine strain on the immune response and protection against tuberculosis. FEMS Microbiol Rev 2008;32:821-41.

[10] World Health Organization. Reported estimates of BCG coverage, http://apps. who.int/immunization_monitoring/globalsummary/timeseries/ tscoveragebcg.html [accessed 09.04.2016] 
[11] Flanagan KL, van Crevel R, Curtis N, Shann F, Levy O, Optimmunize N. Het erologous ("nonspecific") and sex-differential effects of vaccines: epidemiology, clinical trials, and emerging immunologic mechanisms. Clin Infect Dis Official Publ Infect Dis Soc Am 2013;57:283-9.

[12] Ritz N, Mui M, Balloch A, Curtis N. Non-specific effect of Bacille CalmetteGuerin vaccine on the immune response to routine immunisations. Vaccine 2013;31:3098-103.

[13] World Health Organization. WHO Prequalified Vaccines, https://extranet.who. int/gavi/PQ Web/?AspxAutoDetectCookieSupport=1 [accessed 17.08.2016].

[14] Ritz N, Dutta B, Donath S, Casalaz D, Connell TG, Tebruegge M, Robins Browne R, Hanekom WA, Britton WJ, Curtis N. The influence of bacille Calmette-Guerin vaccine strain on the immune response against tuberculosis: a randomized trial. Am J Respir Crit care Med 2012;185:213-22.

[15] Dierig A, Tebruegge M, Krivec U, Heininger U, Ritz N. Paediatric tuberculosis network European trials g. Current status of bacille calmette guerin (BCG) immunisation in Europe - a ptbnet survey and review of current guidelines Vaccine 2015;33:4994-9.

[16] Ritz N, Curtis N. Mapping the global use of different BCG vaccine strains. Tuberculosis 2009;89:248-51.

[17] Marais BJ, Seddon JA, Detjen AK, van der Werf MJ, Grzemska M, Hesseling AC Curtis N, Graham SM, WHO Child TB Subgroup. Interrupted BCG vaccination is a major threat to global child health. Lancet Respir Med 2016;4:251-3.

[18] Tebruegge M, Ritz N, Koetz K, Noguera-Julian A, Seddon JA, Welch SB, Tsolia M, Kampmann B. Availability and use of molecular microbiological and immunological tests for the diagnosis of tuberculosis in europe. Plos One 2014;9:e99129.

[19] Tebruegge M, Salo E, Ritz N, Kampmann B. On behalf of the Paediatric Tuberculosis Network European Trialsgroup P. Inclusion of latent tuberculosis infection as a separate entity into the international classification of diseases. Thorax 2013;68:588.

[20] Clothier HJ, Hosking L, Crawford NW, Russell M, Easton ML, Quinn JA, Buttery JP. Bacillus Calmette-Guerin (BCG) vaccine adverse events in Victoria,
Australia: analysis of reports to an enhanced passive surveillance system. Drug Saf 2015;38:79-86.

[21] Kontturi A, Nohynek H, Salo E. Change in the BCG vaccine strain. Finn Med J 2016;71. Finnish.

[22] BCG Vaccine Shortage. Information bulletin. North Sydney: Ministry of Health, NSW Government; 2016. available from: http://www0.health.nsw.gov.au/ policies/ib/2016/pdf/IB2016_010.pdf [accessed 09.04.2016].

[23] BCG vaccine biomed-lublin. 2016. available from: https://www.mesvaccins. net/web/vaccines/538-vaccin-bcg-biomed-lublin [accessed 09.04.2016].

[24] Krysztopa-Grzybowska K, Brzezinska S, Augustynowicz-Kopec E, Polak M, Augustynowicz E, Lutynska A. Descendant of daughter Brazilian BCG Moreau substrain in Poland. Vaccine 2012;30:5512-8.

[25] Public Health England. Vaccine update: issue 244. Special Edition. April 2016. available from:, https://www.gov.uk/government/publications/vaccine-updateissue-244-april-2016-bcg-supply-special-edition [accessed 20.05.2016].

[26] Favorov M, Ali M, Tursunbayeva A, Aitmagambetova I, Kilgore P, Ismailov S, Chorba T. Comparative tuberculosis (TB) prevention effectiveness in children of Bacillus Calmette-Guerin (BCG) vaccines from different sources. Kazakhstan Plos One 2012;7:e32567.

[27] Abdallah AM, Hill-Cawthorne GA, Otto TD, Coll F, Guerra-Assuncao JA, Gao G, Naeem R, Ansari H, Malas TB, Adroub SA, Verboom T, Ummels R, Zhang H, Panigrahi AK, McNerney R, Brosch R, Clark TG, Behr MA, Bitter W, Pain A. Genomic expression catalogue of a global collection of BCG vaccine strains show evidence for highly diverged metabolic and cell-wall adaptations. Sci Rep 2015;5:15443.

[28] Nieminen T, Salo E. Lymphadenitis in the left groin due to Bacillus CalmetteGuerin (BCG) vaccination. Duodecim 2004;120:2247-50. Finnish.

[29] Salo EP. BCG in Finland: changing from a universal to a selected programme. Euro Surveill 2006;11:18-20.

[30] United Nations Children's Fund. BCG vaccine: current supply \& demand outlook. UNICEF Supply Division; 2015. available from: http://www.unicef. org/supply/files/BCG_Supply_Status_December_2015.pdf. 